\title{
Improvement of the Mass Spectrometry Process on an Ion Implantation
}

\author{
José Lopes ${ }^{1}$, Jorge Rocha², Luís Redondoํㅜ, Francisco Alegria ${ }^{3}$ \\ ${ }^{1}$ Instituto Superior de Engenharia de Lisboa, Rua Conselheiro Emídio Navarro, Lisbon, Portugal \\ ${ }^{2}$ Instituto de Plasmas e Fusão Nuclear, Instituto Superior Técnico, Universidade de Lisboa, Lisbon, Portugal \\ ${ }^{3}$ Instituto Superior Técnico and Instituto de Telecomunicações, Lisbon, Portugal \\ Email: jgabriel@deea.isel.ipl.pt, jroc@ctn.ist.upl.pt, Imredondo@deea.isel.ipl.pt, falegria@lx.it.pt
}

Received 4 February 2016; accepted 6 March 2016; published 9 March 2016

Copyright (C) 2016 by authors and Scientific Research Publishing Inc.

This work is licensed under the Creative Commons Attribution International License (CC BY).

http://creativecommons.org/licenses/by/4.0/

(c) (i) Open Access

\begin{abstract}
Mass spectrometry is an essential part of ion implantation. Therefore, in order to guarantee beam purity avoiding contamination of the implanted samples, a system was developed for the high current implanter at the Laboratory of Accelerators and Radiation Technologies (LATR) at the Instituto Superior Técnico. The system presented and discussed in this paper was developed using a LabVIEW code and uses a PC to control and display the mass spectrum. It also permits to save all data acquired for posterior analysis. In order to show some capabilities of this system, some experimental results are presented.
\end{abstract}

\section{Keywords}

Ion Implanter, LabVIEW, Mass Spectrum

\section{Introduction}

The ion implanter installed at Laboratory of Accelerators and Radiation Technologies (LATR) at the Nuclear and Technological Campus of Instituto Superior Técnico (IST) is dedicated to materials research which makes isotope selection an important requirement. Being the selectivity of the implanted chemical species one of the most relevant advantages of ion implantation, the mass spectrometry is essential in this process in order to guarantee beam purity avoiding the contamination of the implanted samples [1]-[5].

With the initial spectrometer system, the mass spectrum was obtained using a plotter. The operator had to perform all the calculations required for calibration. The system developed and presented in this paper permits to the user to control and analyze the mass spectrometry through a PC application, optimizing the entire mass spectrometry process. This system also allows exporting all data acquired for further analysis. 


\section{System Description}

The high current ion implanter installed at LATR-IST, represented in Figure 1, is the model 1090 of Danfysik [6] [7].

The operating flexibility of the ion source (gas, sputter an oven version), makes possible the production of ion beams from nearly all elements of the periodic table [8] [9]. However, for safety reasons, radioactive or very hazardous elements are not handled.

Being possible to achieve for singly charged ions an energy of $210 \mathrm{keV}$, the maximum ion beam acceleration voltage is $210 \mathrm{kV}$ (160 kV post-acceleration plus on $50 \mathrm{kV}$ extraction). The acceleration tube is specially developed to minimize space charge effects and hence avoid expansion of the beam in an uncontrolled manner. Furthermore, by blocking back streaming electrons it minimizes X-ray emission from the acceleration section. With an electromagnetic two-dimensional beam scanning system the ion beams may be scanned for homogenous exposure over large areas. The maximum beam scanning area depends on the mass-energy product.

One of the components of the ion implanter is an analyzer magnet that bends the ion beam through a right angle to select the desired impurity ions from the output of the source. A charged particle moving with velocity $v$ through a magnet field $B$ will experience a force, given by

$$
F=q \times(v \times B)
$$

where $R$ is the magnet deflection radius and $q$ is the charge. The magnitude of the magnet field may be adjusted to select an ion species with a given mass:

$$
|B|=\sqrt{\frac{2 \times M \times v}{q \times R^{2}}} .
$$

In order to obtain a mass spectrum, two analog signals were necessary to acquire: one proportional to the analyzing magnet magnetic field (x-axes) and another proportional to the beam current intensity (y-axes). The first signal was taken from a voltage output proportional to the analyzing magnet current, available from the magnet power supply. The use of this signal comprised two main difficulties: 1) the analyzing magnet is located at the terminal potential, therefore, an optic-fiber based circuit had to be developed to send the magnet current signal to the ground potential; 2) to avoid magnet hysteresis, it was necessary to continuously increase the magnet the current during a mass spectrum, therefore, another optic-fiber based circuit had to be developed to send the magnet current control signal to the terminal potential. The second signal was taken from the output of the beam stopper, eventually from other beam current measuring device as the Faraday cup or a differential meter

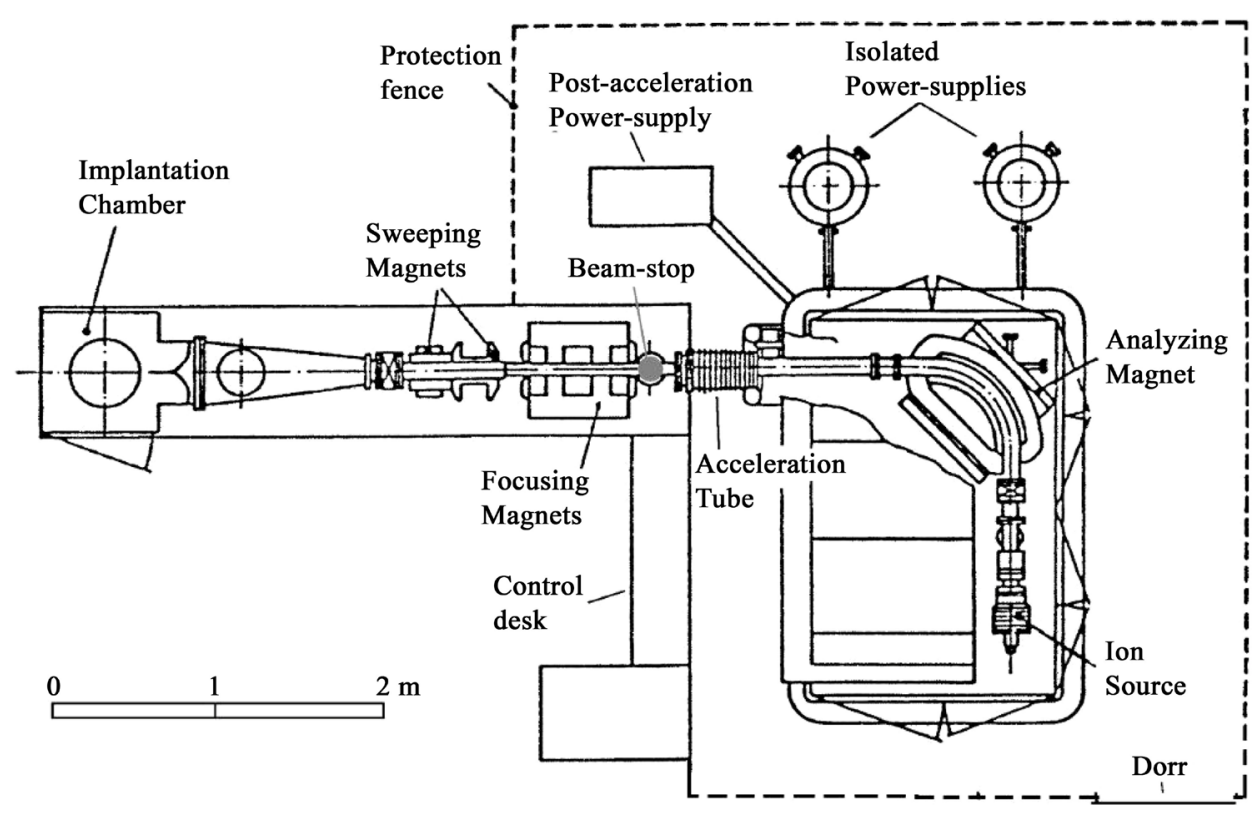

Figure 1. Layout of the high-current ion implanter installed at the LATR. 
[10], located at the ground potential.

The whole system was developed using the LabVIEW program, from National Instruments. To establish the connection between the PC and the ion implanter, a multifunction input/output board from National Instruments, model USB-6251 was used.

The user interface for the develop application is show in Figure 2, where a mass spectrum done before a Manganese (Mn) Implantation, using the oven source version.

Due to the fact that LabVIEW is a graphical programming language specially created for instrumentation and measurement [11] [12], was possible to developed a simple interface using buttons an indicators (numerical and graphical) to control the ion implanter, that can be used without a major experience.

With this application, the user can control the ion implanter through a PC and save and export the data obtained with the mass spectrometry.

As already mentioned, the mass spectrometry is an essential step of ion implantation in order to make a precise selection of the implanted isotope. With this system, the mass spectrometry PC application displays the mass spectrum and offers the possibility to analyze online the results.

For the calibration the user only needs to input the mass values of two different elements apart from each other. Usually is used, as lowest calibration peak, the mass value of Hydrogen, which is always present due to residual moisture inside the ion source, and the support gas used in the ion source, (usually Argon for sputter and oven versions), as highest calibration peak, which is the most intense beam current out of the ion source. Calibration is then done automatically.

For the calibration calculus, and taking in account the describe above, considering the horizontal axis as a reference, the different line points are given by

$$
\text { Calibration }=\sqrt{\frac{M}{q}}
$$

where $M$ is the isotope mass (in a.m.u), $q$ is its charge and the calibration units are arbitrary. After calibration a chart is presented in the user interface screen showing the acquired spectrum with correct mass and beam current values, which allows the user to identify all other peaks present on the spectrum.

The developed application allows for the user to save two files related to the obtained mass spectrum: 1) a txt format file with all the x-y data acquired by the DAQ; 2) a bmp format file with the obtained spectrum. The txt file is extremely important because it will give to the user the ability to view the acquired data on spreadsheet software for more detailed analysis. The bmp file gives to the user the possibility to study or compare mass spectra with the help of a simple design program.

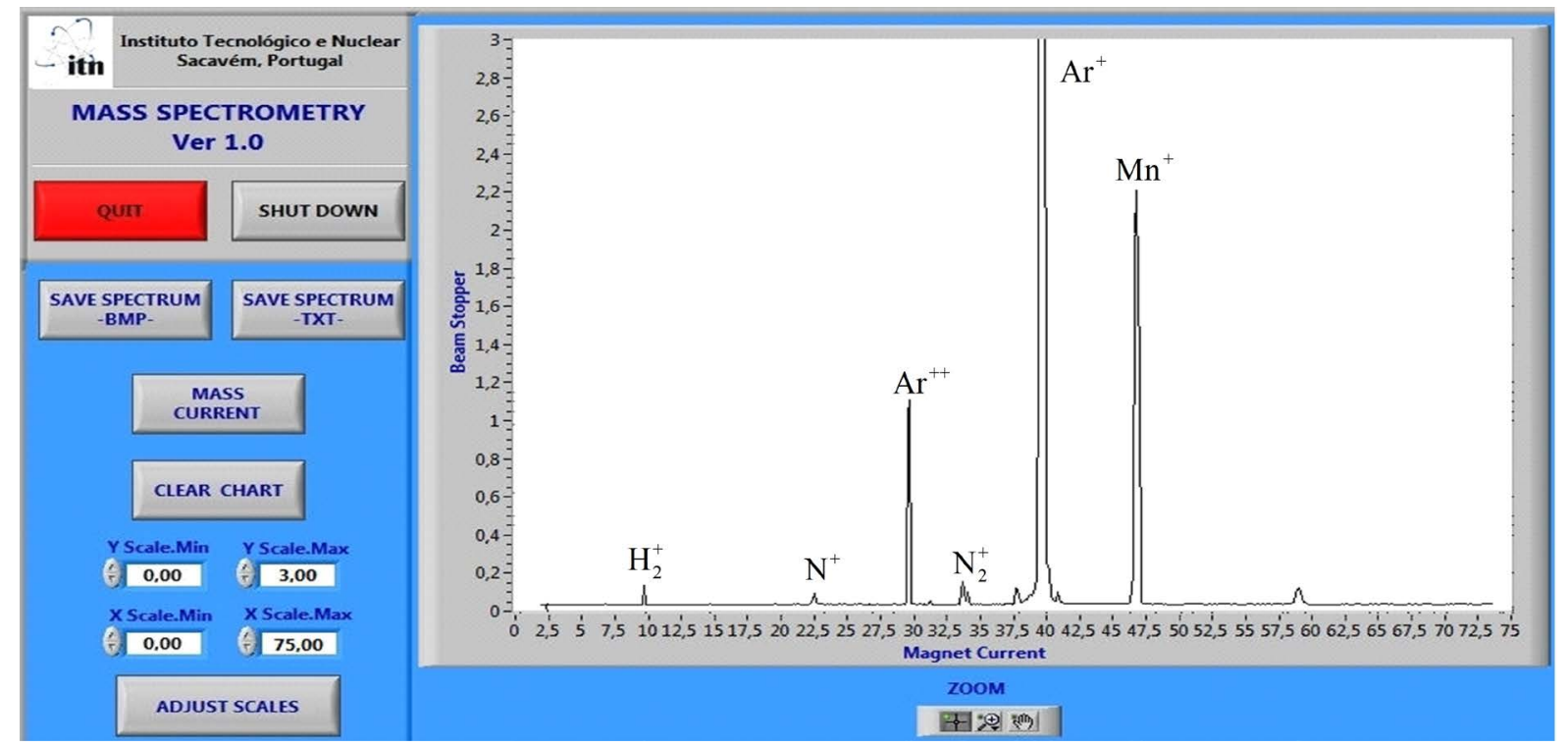

Figure 2. User interface to control and monitoring the application. 
It is also important to mention that the files saved by the application will permit the user to get a more accurate calibration reducing errors when a more accurate definition between isotopes is needed.

\section{Conclusions}

The control system presented in this paper was developed to improve the mass spectrometry of the ion implanter used by LATR-IST for scientific research. This system used a personal computer, one multifunction input/ output board, some custom made electronic interface modules and a LabVIEW application.

One of the goals was to acquire the signals of the ion implanter and give the information to the user through a PC. Currently, the user is able to: 1) observe the mass spectrometry trough the PC; 2) acquire more accurate $x-y$ values; 3 ) automatically calibrate these values more quickly and efficient; 4 ) automatically save all mass spectrometry data for further analysis.

At this time the system is in use and new developments can be made such as the full automation of the mass spectrometry process. For that, the system must be able to calibrate by itself. Another goal to achieve is the automatic identification of the elements in the mass spectrometry so any user can be able to know which element is present in the ion beam.

\section{References}

[1] Ryssel, H. and Ruge, L. (1986) Ion Implantation. John Wiley \& Sons Ltd., Hoboken.

[2] Alves, E., Marques, C., da Silva, R.C., Monteiro, T., Soares, J., McHargue, C., Ononye, L.C. and Allard, L.F. (2003) Structural and Optical Studies of Co and Ti Implanted Sapphire. Nuclear Instruments and Methods in Physics Research Section B, 207, 55-62. http://dx.doi.org/10.1016/S0168-583X(03)00522-6

[3] Marques, C., Alves, E., da Silva, R.C., Silva, M.R. and Stepanov, A.L. (2004) Optical Changes Induced by High Fluence Implantation of Au Ions on Sapphire. Nuclear Instruments and Methods in Physics Research Section B, 218, 139144. http://dx.doi.org/10.1016/j.nimb.2003.12.015

[4] Dearnaley, G. and Grant, W.A. (1976) Ion Implantation of Semiconductors. London.

[5] Mateus, R., Dias, M., Lopes, J., Rocha, J., Catarino, N., Duarte, P., Gomes, R.B., Silva, C., Fernandes, H., Livramento, V., Carvalho, P.A., Alves, E., Hanada, K. and Correia, J.B. (2013) Blistering of W-Ta Composites at Different Irradiation Energies. Journal of Nuclear Materials, 438, S1032-S1035. http://dx.doi.org/10.1016/j.jnucmat.2013.01.225

[6] Miranda, P.A., Chestaa, M.A., Cancinoa, S.A., Moralesa, J.R., Dinatora, M.I., Wachtera, J.A. and Tenreirob, C. (2006) Recent IBA Setup Improvements in Chile. Nuclear Instruments and Methods in Physics Research Section B: Beam Interactions with Materials and Atoms, 248, 150-154. http://dx.doi.org/10.1016/j.nimb.2006.02.006

[7] Nielsen, B.R., Abrahamsen, P. and Eriksen, S. (1989) A Universal High-Current Implanter for Surface Modification of Materials. Materials Science A, 116, 193.

[8] Torp, B., Nielsen, B.R., Rück, D.M., Emig, H., Spädtke, P. and Wolf, B.H. (1990) High-Current Ion Beams of Metallic Elements. Review of Scientific Instruments, 61, 595. http://dx.doi.org/10.1063/1.1141928

[9] Lopes, J. and Rocha, J. (2015) Simple General Purpose Ion Beam Deceleration System Using a Single Electrode Lens. World Journal of Engineering and Technology, 3, 127-133. http://dx.doi.org/10.4236/wjet.2015.33014

[10] Redondo, L.M., Rocha, J. and Soares, J.C. (2007) A Low-Cost, Accurate and Non-Intercepting Continuous Method Forbeam Current Measurements in a High-Current Ion Implanter. Nuclear Instruments and Methods in Physics Research B, 265, 576-580. http://dx.doi.org/10.1016/j.nimb.2007.09.042

[11] Lopes, J., Rocha, J., Redondo, L. and Alegria, F. (2011) High Resolution Ion Beam Profile Measurement System. Proceedings of ICALEPCS2011, Grenoble, MOPKS003.

[12] Havránek, V., Hnatowicz, V., Macková, A. and Voseček, V. (2009) Laboratory of Nuclear Analytical Methods of Nuclear Physics Institute ASCR. International Topical Meeting on Nuclear Research Applications and Utilization of Accelerators, AP/P5-06, Vienna, 4-8 May 2009. 